\title{
FLUCTUATIONS OF THE DIFFUSE GALACTIC BACKGROUND
}

\author{
K. Mattila \\ Helsinki University Observatory \\ Tähtitorninmäki \\ SF-00130 Helsinki, Finland
}

An analysis of fluctuations in the brightness of the Milky Way using the concept that interstellar matter occurs in the form of discrete clouds was first applied by Ambarzumian (1940, 1944). This theory was formulated in a general way and discussed in great detail in a series of papers by Chandrasekhar and Münch (1950a, 1950b, 1951, 1952), by Münch and Chandrasekhar (1952), and by Limber (1953). More recently Peters (1970) presented an analysis of this kind based on extensive photographic observational material. Although the influence of clumpiness of the dust distribution on the mean integrated starlight was thoroughly discussed in these papers, it has not been properly included in most of the photometric models of the Galaxy. Only the models of Caplan and Grec (1979) and Mattila (1980a, 1980b) incorporate these effects.

In these investigations, the surface brightness fluctuations were considered as being due solely to integrated starlight, which is modulated by purely absorbing dust clouds. But the diffuse galactic light (DGL), i.e., the light scattered by the clouds, will also show fluctuations, and it contributes to the fluctuations of the total Milky Way brightness. This concept is important both for the interpretation of the mean intensity of the DGL (Mattila, 1971) and for the analysis of the diffuse and total Milky Way brightness fluctuations (Mattila and Scheffler, 1978).

An analysis of the infrared background radiation fluctuations along the lines described above for the optical would be very timely and rewarding with the IRAS data set. Data sufficiently detailed to motivate a statistical analysis may also soon become available. It is interesting to note that Jakobsen and Kahn (1986), who modeled the galactic soft X-ray background in terms of absorbing clouds being interspersed randomly within the emitting hot gas, rediscovered the Chandrasekhar and Münch formulae.

\section{REFERENCES}

Ambarzumian, V.A. 1940, Bull. Abastumani Astrophys. Obs., 4, 17.

Ambarzumian, V.A. 1944, Compt. Rend. (Doklady) Acad. Sci. USSR, 14, 223.

Caplan, J., Grec, G. 1979, Astron. Astrophys., 78, 335.

Chandrasekhar, S., Münch, G. 1950a, Astrophys. J., 112, 380.

Chandrasekhar, S., Münch, G. 1950b, Astrophys. J., 112, 393.

Chandrasekhar, S., Münch, G. 1951, Astrophys. J., 114, 110.

Chandrasekhar, S., Münch, G. 1952, Astrophys. J., 115, 103.

Jakobsen, P., Kahn, S.M. 1986, Astrophys. J., 309, 682.

Limber, D.N. 1953, Astrophys. J., 117, 145.

Mattila, K. 1971, Astron. Astrophys., 15, 292.

Mattila, K. 1980a, Astron. Astrophys. Suppl. Ser., 39, 53.

Mattila, K. 1980b, Astron. Astrophys., 82, 373.

Mattila, K., Scheffler, H. 1978, Astron. Astrophys., 66, 211.

Münch, G., Chandrasekhar, S. 1952, Astrophys. J., 115, 94.

Peters, G. 1970, Astron. Astrophys., 4, 134. 